\title{
Hybrid Artificial Neural Networks Based Models for Electricity Spot Price Forecasting - A Review
}

\author{
Fan Zhang \\ Dalarna University \\ Departments of Energy Technology and Computer \\ Engineering \\ Falun, 79188, Sweden \\ fzh@du.se
}

\author{
Hasan Fleyeh \\ Dalarna University \\ Department of Computer Engineering \\ Falun, 79188, Sweden \\ hfl@du.se
}

\begin{abstract}
Electricity price forecasting plays a crucial role in a liberalized electricity market. In terms of forecasting approaches, artificial neural networks are the most popular among researchers due to their flexibility and efficiency in handling complexity and non-linearity. On the other hand, a single neural network presents certain limitations. Therefore, in recent years, hybrid models that combine multiple algorithms to balance out the advantages of a single model have become a trend. However, a review of recent applications of hybrid neural networks based models with respect to electricity price forecasting is not found in the literature and hence, the motivation of this paper is to fill this research gap. In this study, methodologies of existing forecasting approaches are briefly summarized, followed by reviews of neural network based hybrid models concerning electricity forecasting from year 2015 onwards. Major contributions of each study, datasets adopted in experiments as well as the corresponding experiment results are analyzed. Apart from the review of existing studies, the novelty and advantages of each type of hybrid model are discussed in detail. Scope of the review is the application of hybrid neural network models. It is found that the forecast horizon of the reviewed literature is either hour ahead or day ahead. Medium and long term forecasting are not comprehensively studied. In addition, though hybrid models require relatively large computational time, time measurements are not reported in any of the reviewed literature.
\end{abstract}

Keywords--Electricity price forecasting, neural networks, electricity markets, computational intelligence, machine learning.

\section{INTRODUCTION}

As a result of the first two directives of the European Union's electricity markets decades ago, transmission and distribution networks are accessible to third parties and independent regulatory agencies are introduced. In addition, domestic and industrial consumers are free to choose electricity suppliers. The third directive was adopted in 2009, which further enhanced the liberalization of the internal electricity market [1]. The objective of the electricity market liberalization is to open electricity markets, lower electricity prices, and promote competition and efficiency [2]. Accurate price forecasting results can be utilized by stakeholders to minimize the cost, mitigate potential risks as well as fulfill environment policy goals [3]. Therefore, electricity price forecasting plays an increasingly important role in a liberalized and deregulated market.

In general, electricity price forecasting can be categorized into short, medium and long-term according to the forecasting horizons. To be more specific, short term forecasting generally forecasts the price from minutes to days ahead, such forecasting plays an important role in daily market operations. According to short-term forecasting result, market players decide the timing of purchasing or selling to gain maximized profit [4]. Medium-term time horizons are from days to months ahead. Risk management, derivative marketing pricing and bilateral contracting are common application areas of mediumterm forecasting. In terms of long-term electricity forecasting, its time frame ranges from months to years. Long-term electricity price forecasting is useful for investment profitability analysis as well as grid or transmission expansion planning [5].

Existing electricity price forecasting approaches include multi-agent, fundamental, statistical, reduced-form and computational intelligence models, among which artificial neural network (ANN) models are regarded as the state of art approach [6]. To be more specific, artificial neurons are connected together to form an artificial neural network. Synaptic weights are assigned to each connection. These weights store and update the knowledge learned that enable ANN to mimic the behavior of the human brain. On the other hand, a single ANN model presents certain limitations. For example, without an effective supplementary optimization algorithm for parameter tuning, optimal performance of a single ANN is not guaranteed. Therefore, hybrid models have become a trend in recent years.

Neural networks based hybrid models are models that consist of neural networks and other machine learning algorithms [7]. For example, evolutionary algorithms (EAs) are commonly used in hybrid models for parameter 
optimization. EAs are generic population-based metaheuristic optimization algorithms inspired by biological evolution. General processes involved in EAs are: representation of a solution by initialized individuals, evaluation of fitness value in each iteration and new population generation [8]. Some popular EAs among researchers are genetic algorithm (GA) [9], particle swarm optimization (PSO) [10] and differential evolution (DE) [11].

However, the latest studies from 2015 are not covered in the existed reviews [12], [6]. In addition, technical details, application scenarios, advantages and disadvantages of different hybrid approaches are not well discussed in the literature, although hybrid models have become the trends. Therefore, the motivation of this paper is to fill this research gap and pave the path for future researchers in this field.

The major contribution of this paper is to perform a review of the existing approaches and summarize the advantages and disadvantages of each approach. It is performed by an exhaustive review of various hybrid ANN based approaches from year 2015 onwards and performing a comparative analysis for each hybrid ANN model, as well as analyzing the future trend that inspires researchers to further explore this field. The scope of this study is limited to the applications of ANN based hybrid models.

The rest of this paper is structured as follows: in Section II, existing approaches of electricity price forecasting are reviewed, followed by a short overview of ANN model. Applications of ANN based hybrid models are reviewed in Section III. Discussions and analysis of each hybrid model are presented in Section IV. Conclusion of this paper is provided in Section V.

\section{METHODOLOGY}

\section{A. Overview of existing approaches}

Taxonomy of electricity price approaches presented in different literature varies. The presented taxonomy in Fig. 1 is modified based on [6] and is used in this article. Due to the increasing popularity of ANN based models and their superiority in performance compared with other non-ANN based models, the forecasting approaches are divided into ANN based and non-ANN based models accordingly.

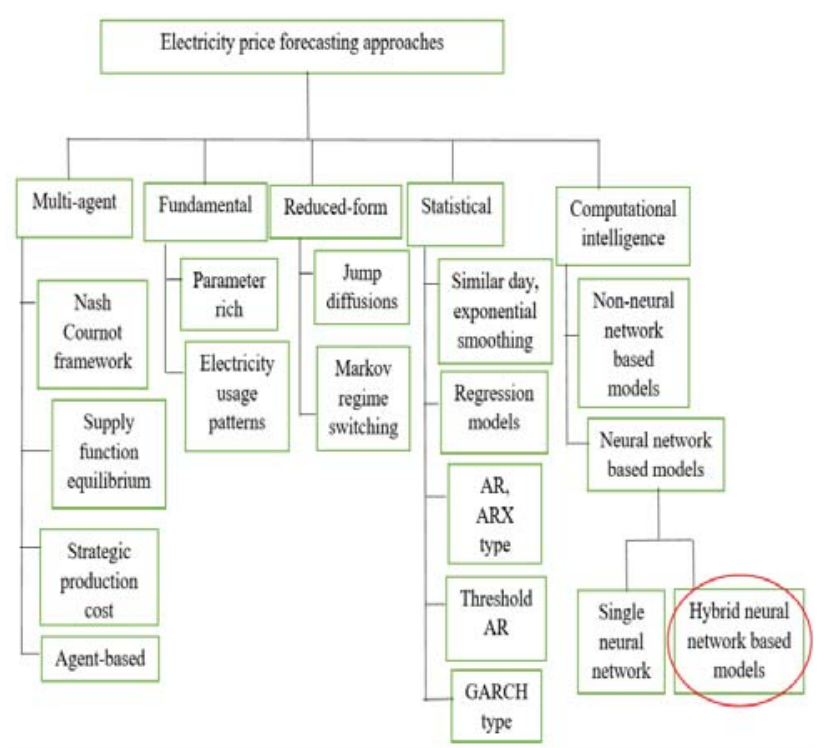

Fig. 1. Electricity price forecasting approaches

There are five major approaches to electricity price forecasting:

- Multi-agent models: These models simulate the interactions between heterogeneous agents and the environment. To be more specific, agents react to the changing environment, such as supply and demand dynamics, other competitors' strategic decisions, etc. based on the learned past experience and forecast the price accordingly [5]. By incorporating the dynamic changes in electricity markets, agent based models are flexible in strategic decision-making. However, defining the environment, the way agents interact with each other, etc. is challenging.

- $\quad$ Fundamental models: These models make the assumption of relationships between fundamental drivers, such as temperature, demand patterns, plant costs, etc. These physical and economic factors are important inputs when modeling price dynamics [13]. Historic data of observable fundamental inputs can be utilized as a new set of training data for such models. Other important factors, such as temperature measurements can be utilized to calibrate model parameters by fundamental modeling approach. However, violations of the assumptions lead to unreliable forecast results. Besides, the effort of determining model parameters depends on the detail level of a fundamental model.

Reduced-form models: they are suitable for modeling the stochastic behavior of electricity price. Typical stochastic factors are jump diffusion, regime switching, meanreversion [14]. Such models perform well in electricity price spike and volatility forecasting and are relatively simple. However, reduced-form models are mostly applied for simulating major characteristics of daily electricity price instead of price forecasting.

Statistical models: they are represented by mathematical combinations of previous prices and other exogenous factors, such as weather conditions and consumption patterns. Interpretability is the major advantage of statistical models. Besides, important fundamental factors such as historical demand, fuel price, forecasts of weather and 
consumptions can be incorporated into the model. However, performance degrades when modeling non-linearity or spikes present in the electricity price spikes need to be filtered and replaced with normal values in such case [15].

\section{-}

Computational Intelligence models: definition

of computational intelligence models varies in literature. In general, computational intelligence models utilize different artificial intelligence techniques such as machine learning and soft computation algorithms. Among all computational intelligence models, ANNs are most widely used with respect to electricity price forecasting [16].

\section{REVIEW OF THE HYBRID MODELS}

\section{A. $\quad$ Model $1(K-N N+A N N)$}

This model combines K-Nearest Neighbor (KNN) with Artificial Neural Network (ANN). KNN algorithm is utilized for selecting relevant input and reducing the size of training data, aiming to improve the performance of a single ANN. The capability of modeling the relationship between input and output of each subset as well as the dependencies between input variables of a subset is the major Advantage of k-NN algorithm.

As an application of this model in 2016, the European Power Exchange (EPEX) day ahead price forecasting was proposed. The proposed model was tested for the period from January $1^{\text {st }}, 2013$ to September $30^{\text {th }}, 2013$. The average RMSE of the proposed model was 6.58. An important finding reported in this study is that the electricity price was positively correlated with the price of the same hour one day before. Thus, instead of using the latest forecasted price as input for forecasting next step, electricity price of the same hour one day before is used, which eliminates the accumulated errors of iterative approach and the complexity of direct approach [17].

\section{B. Model 2 (Ensemble ANNs)}

Ensemble multilayer perceptron neural networks (MLPNNs) is capable of modeling more complex electricity prices than a single MLPNN. In order to boost the learning ability of hybrid multiple MLPNNs, one scientific approach is utilizing different and appropriate learning algorithms for each single MLPNN based on the characteristic of the problem to be solved. A scientific combination of different ANNs also improves the model performance, e.g. while Radial Basis Functions Neural Networks (RBFNN) is suitable for modeling the local characteristics of electricity prices, MLPNN works well for capturing the global trend of electricity prices. Therefore, combing both together improve the learning ability compared with a single MLPNN or RBFNN.

As a study of this hybrid approach, an hourly day ahead electricity forecasting of Nord Pool market was proposed in 2018. The hybrid model consisted of three MLPNNs with different learning algorithms: Levenberg-Marquardt (LM), Levenberg-Marquardt (BFGS) and Bayesian Regularization (BR). Historical electricity price and wind power data were used as input features. Forecasting result of four days from four seasons in 2012 was presented, the average mean absolute percentage error (MAPE) of the proposed method was 2.94 .
Another finding of this study is that the forecasting result is improved by incorporating wind power generation factor [18].

\section{Model 3 (Fuzzy logic + ANN)}

This model combines Fuzzy logic and ANN to perform the forecasting. Fuzzy logic is capable of transforming linguistic information into numeric data, the defuzzfication result can be served as the input to the ANN model. Another advantage of combining fuzzy logic is the capability of softening high frequency changes of series data, which is commonly observed in the electricity price series. As an application of this hybrid approach, an asymmetric Gaussian fuzzy neural network (AGFINN) was proposed for day ahead electricity price forecasting in 2017. The proposed asymmetric neuro fuzzy system is derived from existing Takagi-Sugeno-Kang (TSK) fuzzy systems [19] by replacing the IF part of fuzzy rules with an asymmetric Gaussian function. Moreover, unlike the adaptive network based fuzzy inference system (ANFIS), the proposed AGFINN reduces the number of fuzzy rules by utilizing a fuzzy c-means clustering technique to avoid the high dimensionality problem. Data of independent system operator (ISO) New England market from 2006 to 2007 was employed in the case study. Different combinations of lagged price and load were selected as the input features. In terms of the forecasting result, the least MAPE of the proposed method was 4.24. Result also shows the proposed approach outperforms alternative models: Adaptive Fuzzy Logic System (AFLS), ANFIS and MLP considered in this study [20].

\section{Model $4(E A+A N N)$}

Evolutionary Algorithms (EA) can be invoked to improve the performance of neural networks by avoiding getting into local minima. By utilizing the stochastic/non-monotonic search ability of evolutionary algorithms which tackles the local minima problem, and enables the neural network to achieve faster convergence.

As an application of this model, an extreme learning machine (ELM) based hybrid approach was proposed for electricity price forecasting of the Australian energy market in 2016. In this study, a new mutation strategy is proposed for DE algorithm which achieves a tradeoff between local exploitation and global exploration and faster convergence. Australian energy market operator (AEMO) data ranging from June 1st, 2006 to May 31st, 2007 was employed in the case study. Two weeks' data prior to the forecasting day was used for training, forecasting result of seven days from each season was presented. Testing MAPE of the proposed model is 6.08, 9.20, 21.14 and 11.95 for winter, spring, summer and autumn week respectively [21].

\section{E. Model 5 (Hybrid deep neural networks)}

This model employs a hybrid deep learning neural Network (DNNs). In general, the learning ability of deep neural networks is superior to shallow neural networks, a combination of various deep neural network layers in a scientific way further enhances the model performance.

One example application of this hybrid approach in 2018 is the utilization of long short-term memory (LSTM) DNN and gated recurrent units (GRU) DNN for the day ahead electricity spot price forecasting. The motivation of LSTM-DNN was to 
include both the recurrent layer and regular layer for modeling relations inside the sequential time series data and nonsequential data. In terms of GRU-DNN. A GRU layer, which is faster for training, was used for modeling the time sequence. EPEX-Belgium market data was employed in the case study. Training set consists of data from January 1st, 2010 to November 30th, 2014, data from December 1st, 2014 to November 30th, 2015 was used for validation and rest of the data was for testing. SMAPE of LSTM-DNN and GRU-DNN were 13.06, 13.04 respectively [22].

Another example is the application of a hybrid structured DNN that consisted of two different types of layers: the convolutional neural network $(\mathrm{CNN})$ and LSTM. In the first step, CNN was used to extract the features. Then extracted features were fed to LSTM for forecasting. Model input was historic electricity price of 24 hours and output was the forecasted price of next hour. PJM Regulation Zone Preliminary Billing Data which was composed of regulation market capacity clearing price of every half hour in 2017 was employed in the study. Average mean absolute error (MAE) of the proposed hybrid model was 8.85 which was lower than a single LSTM, CNN model [23]. Although DNNs were utilized in both studies, the major difference lied in the types of DNNs adopted.

\section{F. Model $6(E A+$ Wavelet + ARMA + ANN $)$}

This model combines evolutionary algorithms, wavelets, autoregressive moving average (ARMA), and artificial neural networks to achieve the price forecast. Wavelet transformation decomposes the original electricity prices data into a set of well-behaved sub-series that is easier for prediction. ARMA model is utilized to model the linear part of electricity prices while RBFNN is used for capturing non-linear patterns of the residual. PSO is applied to optimize the number of hidden nodes of RBFNN as well as the parameters of radial basis function, namely, center and spread.

As one application of this model in 2017, a hybrid approach consisted of Wavelet transformation, self-adaptive PSO, kernel extreme learning machine (KELM) [24] and ARMA was presented for short term electricity price forecasting. The overall process of the proposed approach is: Firstly, the Wavelet transformation of original series data was performed. Based on the decomposition result, ARMA and KELM were used to forecast both linear and non-linear part of the series data. Besides, the self-adaptive PSO algorithm was utilized to optimize the penalty factor and kernel parameters of KELM. Data of PJM, Australian and Spanish market was employed in the case studies. The least average MAPE of the proposed method was 7.66 and 5.23 for PJM 2006 and 2004. In terms of the Australian market, the least MAPE was 1.51 and 2.34 for year 2014 and 2013. The reported least MAPE for the Spanish market was 3.6 and 1.86 for year 2009 and 2015 respectively [25].

\section{G. $\quad$ Model $7(S O M+A N N)$}

Self-Organizing Maps (SOM)are useful for partitioning electricity price data into clusters according to the similarity of dynamic properties, and each cluster is an approximately stationary series. As a result, ANN performs better using the pre-processed data by SOM.
As a study of this model, a hybrid SOM, ANN approach was proposed for day ahead electricity price forecasting in 2015. The proposed approach consisted of two steps: first, time series clustering was performed to model pattern sequence and corresponding topology relations by SOM and the pattern sequence was labeled using its topological coordinates. Then, ANN was utilized to model the relation between a pattern sequence of a certain length and the topology coordinates of its next pattern. Forecasting results of Spanish market 2002 and New York market 2010 were presented. MER of the proposed model was 3.96, 3.59, 5.32 and 6.18 for winter, spring, summer and autumn week. In terms of the result of the New York market, MAPE of the proposed approach was 3.7, 3.44, 2.39 and 2.33 for winter, spring, summer and autumn week respectively [26].

\section{H. Model $8(E A+$ Wavelet $+A N N)$}

This model combines Evolutionary Algorithms, Wavelet transforms and Artificial Neural Network electricity price forecast. Wavelet transformation is widely used for decomposing ill-behaved electricity prices data into a set of constitutive series with less variance and outliers, which improve the forecasting accuracy of ANN. Besides, EA can be utilized to search for the optimal parameters of ANN, as well as Wavelet, such as the selection of mother Wavelet and level of decomposition.

Three examples of this approach are: the application of a hybrid model consisted of improved environment adaptation method algorithm (IEAM) [27], generalized neuron model [28] and Wavelet transformation for short term electricity price forecasting in 2017. Origin series data was transformed by Wavelet transformation into subseries first. Then generalized neuron models were trained separately using the subseries. Compared with traditional ANNs, generalized neuron model is more flexible when dealing with nonlinearity by using both sum and product as an aggregation function. Meanwhile, IEAM with improved adaptation operator was utilized to optimize the free parameters. Data of New South Wales, Australia (NSW) electricity market was employed in the case study. MAPE of the proposed was 4.84, 0.93, 1.65 and 1.20 for summer, autumn, winter and spring respectively [29].

The application of a hybrid model that combines Wavelet transformation and PSO with ANN for the day ahead MCP forecasting in 2017. To be more specific, first, Wavelet transformation was performed to smooth the origin series by removing its high frequency components. Then, ANN was trained using the smoothed series, meanwhile, PSO was utilized to optimize the weights of ANN. Hourly purchase bid and MCP data of Indian Energy Exchange (IEX) 2014 was employed in the case study. The minimal MAPE of the proposed method was 9.25 [30].

In addition, the application of a hybrid approach consists of Wavelet transformation, Bat algorithm (BA) [31] and ANN model for 24 hours ahead electricity price forecasting in 2018. Wavelet transformation was performed to transform the origin time series into subseries with fewer outliers and more stable variance. Then, based on the decomposed subseries, ANN was used for forecasting. Meanwhile, BA was utilized to optimize the selection of mother wavelet, the level of decomposition, 
number of ANN layers and activation function of the output layer. Forecasting result of Spanish market 2002 and PJM 2006 was presented. For the Spanish market, MAPE of the proposed method was $0.61,1.22,1.30$ and 1.81 for winter, spring, summer and autumn week. While for PJM, MAPE by the proposed model was $0.79,0.80,0.81$ and 0.54 for winter, spring, summer and autumn week [32].The main difference between the three studies are the chosen EAs for optimization.

\section{DISCUSSION}

In general, in a hybrid model, one model is utilized to alleviate another model's disadvantages or optimize the parameters of the other model. Another common hybrid approach is combining models of different characteristics together to bring out the best from all of them [33]. However, there is not a golden rule of designing a hybrid model and hybrid algorithms evolved in recent years.

Two types of hybrid approaches were proposed in several studies from 2015 and 2016: a combination of clustering algorithms with ANNs and combing a single EA with ANN. These two types are relatively easy to implement. However, there are a few drawbacks. To be more specific, in terms of the most commonly applied K-NN algorithm, the hyper parameter $\mathrm{K}$ needs to be carefully pre-specified. On the other hand, the single EA with ANN approach was improved by adding Wavelet transformation to the hybrid model. In fact, such improved approaches with respect to electricity price forecasting were popular among researchers in 2017 and 2018. Major enhancement made by this approach is the utilization of Wavelet transformation that decomposes original electricity prices data into a set of well-behaved sub-series which is easier for prediction. Another variation of the Wavelet EA ANN hybrid model is Wavelet EA ARMA ANN. The motivation of adding ARMA to the hybrid model is that ARMA is proved to be efficient in modeling the linear part of electricity prices. However, the results of hybrid models with EA algorithms are not necessarily reproducible or globally optimal. Therefore, justification of the result is difficult.

In 2017, a combination of fuzzy and ANN was proposed. Though fuzzy c-means algorithm was adopted to reduce the number of fuzzy rules, the task of deriving fuzzy rules itself is non-trivial.

The aforementioned hybrid models fall into the category of shallow neural networks based hybrid models. With the development of parallel-processing techniques, applications of deep neural networks become practical. In general, deep neural networks outperform shallow neural networks for complex problems. Due to the aforementioned factors, the hybrid deep neural network became the focus of most recent studies. It is worth noting that the accuracy of proposed hybrid deep neural networks is higher than models considered in the other literature from 2018.

The number of studies for each hybrid approach from year 2015 to 2018 is shown in Fig.2. It shows that the applications based on DNNs are most popular among researchers in recent year. In addition, among all hybrid models in the reviewed literature, EA, Wavelet combined with ANN is the most commonly adopted approach by researchers.

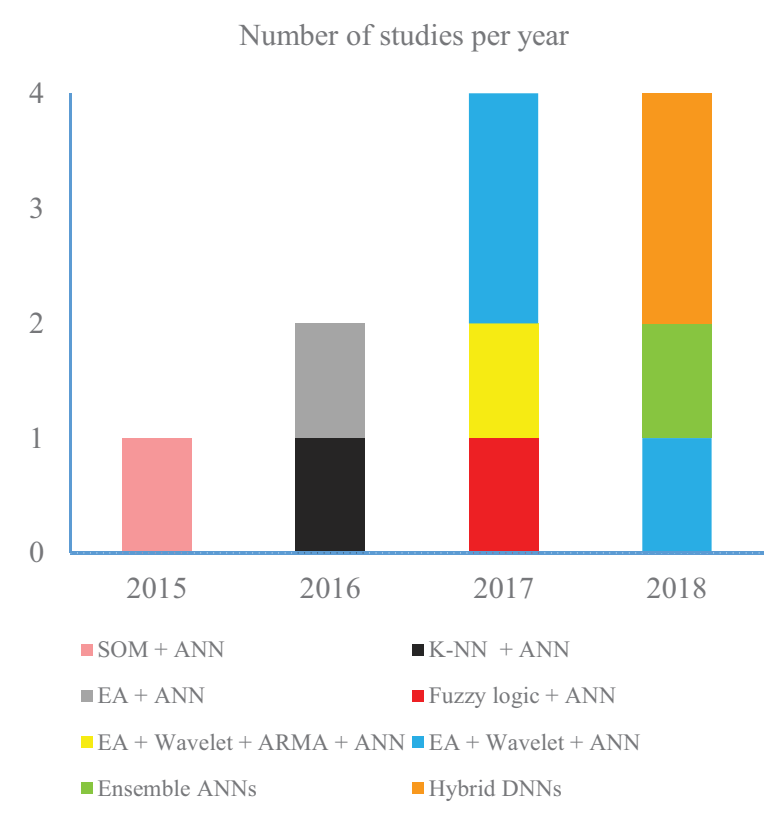

Fig. 2. Number of studies for each hybrid model per year

\section{CONCLUSIONS}

This study provided a review of ANNs based hybrid models for electricity price forecasting. Such forecasting plays an increasingly crucial role in today's liberalized market in terms of decision making. Electricity price data usually present chaotic dynamics, such as non-stationary, unusual spikes, high nonlinearity, etc. In such cases, a single neural network is generally not able to model such complexities well. Therefore, the utilization of hybrid models can be beneficial. In general, by combing various models scientifically, hybrid models become more robust and capable of dealing with problems that are complex in nature than a single model can. Although hybrid models outperform a single ANN model in most cases, drawbacks of hybrid models are increased model complexity, higher computational cost and less interpretability. Besides, optimality of hybrid models is not always guaranteed, especially when stochastic optimization algorithms are involved in the hybrid models. Another finding is that deep neural networks become a trend in recent researches. Deep neural networks have been proved to be superior to shallow neural networks when dealing with more complex real world problems such as long term time series forecasting. However, deep neural networks have not been comprehensively studied in this field. In addition, a comparison between deep neural networks and hybrid models with regard to electricity price forecasting is barely found in the existing literature and is thus a wide research space to be studied. Moreover, though a fast response is essential in real time application and building a hybrid model is relatively time-consuming. Time measurements are not reported in any existing literature, which can be considered in future studies. Another finding is that only short term forecasting the reviewed literature, mid and long term forecasting are not comprehensively studied by researchers. 


\section{REFERENCES}

[1] P. M. Barnes, The Politics of Nuclear Energy in the European Union: Framing the Discourse. Barbara Budrich Publishers, 2017.

[2] L. M. Rathke, M. J. Arentsen, and L. M. Rathke, "The Effects of Electricity Market Liberalisation in the European Union," 2015.

[3] S. Pezzutto, G. Grilli, S. Zambotti, and S. Dunjic, "Forecasting Electricity Market Price for End Users in EU28 until 2020-Main Factors of Influence," Energies, vol. 11, no. 6, pp. 1-18, 2018.

[4] S. Girish, G.P., Vijayalakshmi, "Role of energy exchanges for power trading in India,” Int. J. Energy Econ. Policy, vol. 5(3), pp. 673-676, 2015.

[5] M. Ventosa, M., Baíllo, Á., Ramos, A., \& Rivier, "Electricity market modeling trends," Energy Policy, vol. 33(7), pp. 897-913, 2005.

[6] R. Weron, "Electricity price forecasting: A review of the state-ofthe-art with a look into the future," Int. J. Forecast., vol. 30, no. 4, pp. 1030-1081, 2014.

[7] C. Deb, F. Zhang, J. Yang, S. Eang, and K. Wei, "A review on time series forecasting techniques for building energy consumption," Renew. Sustain. Energy Rev., vol. 74, no. February, pp. 902-924, 2017.

[8] V. Kachitvichyanukul, "Comparison of Three Evolutionary Algorithms : GA, PSO, and DE,”Ind. Eng. Manag. Syst., vol. 11, no. 3, pp. 215-223, 2012.

[9] A. Arabali, M. Ghofrani, M. S. Fadali, and Y. Baghzouz, "GeneticAlgorithm-Based Optimization Approach for Energy Management," vol. 28 , no. 1 , pp. $162-170,2013$.

[10] H. M. I. Pousinho, V. M. F. Mendes, and J. P. S. Catalão, "Shortterm electricity prices forecasting in a competitive market by a hybrid PSO-ANFIS approach," Int. J. Electr. Power Energy Syst., vol. 39, no. 1, pp. 29-35, 2012.

[11] F. Zhang, C. Deb, S. E. Lee, J. Yang, and K. W. Shah, "Time series forecasting for building energy consumption using weighted Support Vector Regression with differential evolution optimization technique," Energy Build., vol. 126, pp. 94-103, 2016.

[12] S. Vijayalakshmi and G. P. Girish, "Artificial neural networks for spot electricity price forecasting: A review," Int. J. Energy Econ. Policy, vol. 5, no. 4, pp. 1092-1097, 2015.

[13] G. Burger, M.; Graeber, B.; Schindlmayr, Managing Energy Risk: An Integrated View on Power and Other Energy Markets. Hoboken, NJ, USA: John Wiley \& Sons, Inc., 2012.

[14] P. Spodniak and V. Bertsch, "Determinants of Power Spreads in Electricity Futures Markets: A Multinational Analysis," no. December, pp. 1-31, 2017.

[15] A. R. H. Geman, "Understanding the fine structure of electricity prices," J. Bus., vol. 79, pp. 1225-1261, 2006.

[16] A. K. S.K. Aggarwal, L.M. Saini, "Electricity price forecasting in deregulated markets: A review and evaluation," Int. J. Electr. Power Energy Syst., vol. 31, pp. 13-22, 2009.

[17] D. Keles, J. Scelle, F. Paraschiv, and W. Fichtner, "Extended forecast methods for day-ahead electricity spot prices applying artificial neural networks," Appl. Energy, vol. 162, pp. 218-230, 2016.

[18] A. Aghajani, R. Kazemzadeh, and A. Ebrahimi, "Short-term prediction of market-clearing price of electricity in the presence of wind power plants by a hybrid intelligent system," Neural Comput. Appl., vol. 8, pp. 1-13, 2018.

[19] B. Rezaee and M. H. F. Zarandi, "Data-driven fuzzy modeling for Takagi-Sugeno-Kang fuzzy system," Inf. Sci. (Ny)., vol. 180, no. 2, pp. 241-255, 2010.

[20] A. Alshejari and V. S. Kodogiannis, "Short-Term Electricity Price Forecasting using Asymmetric Fuzzy Neural Network Systems," 2017.

[21] C. Xiao, Z. Dong, Y. Xu, K. Meng, X. Zhou, and X. Zhang, "Rational and self-adaptive evolutionary extreme learning machine for electricity price forecast," Memetic Comput., vol. 8, no. 3, pp. 223-233, 2016.

[22] J. Lago, F. De Ridder, P. Vrancx, and B. De Schutter, "Forecasting day-ahead electricity prices in Europe: The importance of considering market integration," Appl. Energy, vol. 211, no. July 2017, pp. 890-903, 2018.

[23] P.-H. Kuo and C.-J. Huang, “An Electricity Price Forecasting Model by Hybrid Structured Deep Neural Networks," Sustainability, vol. 10, no. 4, p. 1280, 2018.

[24] A. Iosifidis, A. Tefas, and I. Pitas, "On the kernel Extreme Learning Machine classifier s," Pattern Recognit. Lett., vol. 54, pp. 11-17, 2015.

[25] Z. Yang, L. Ce, and L. Lian, "Electricity price forecasting by a hybrid model, combining wavelet transform, ARMA and kernelbased extreme learning machine methods," Appl. Energy, vol. 190, pp. 291-305, 2017.

[26] C. H. Jin et al., "A SOM clustering pattern sequence-based next symbol prediction method for day-ahead direct electricity load and price forecasting," Energy Convers. Manag., vol. 90, pp. 84-92, 2015.

[27] M. A. Mishra KK, Tiwari S, "A bio inspired algorithm for solving optimization problems," 2011.

[28] D. K. Chaturvedi, M. Mohan, R. K. Singh, and P. K. Kalra, "Improved generalized neuron model for short-term load forecasting," vol. 8, pp. 370-379, 2004.

[29] N. Singh, S. R. Mohanty, and R. Dev Shukla "Short term electricity price forecast based on environmentally adapted generalized neuron," Energy, vol. 125, pp. 127-139, 2017.

[30] S. E. Peter and I. J. Raglend, "Sequential wavelet-ANN with embedded ANN-PSO hybrid electricity price forecasting model for Indian energy exchange," Neural Comput. Appl., vol. 28, no. 8, pp. 2277-2292, 2017.

[31] X. Yang, "Bat algorithm : a novel approach for global engineering optimization," 2011.

[32] P. M. R. Bento, J. A. N. Pombo, M. R. A. Calado, and S. J. P. S. Mariano, "A bat optimized neural network and wavelet transform approach for short-term price forecasting," Appl. Energy, vol. 210, no. October 2017, pp. 88-97, 2018.

[33] M. Wozniak, "Hybrid Classifiers: Methods of Data, Knowledge, and Classifi er Combination," Stud. Comput. Intell. Springer, vol. 519, 2014. 